\title{
Extrahepatic biliary atresia: preoperative assessment and surgical results in 47 consecutive cases
}

\author{
H T PSACHAROPOULOS, E R HOWARD, B PORTMANN, AND A P MOWAT \\ Department of Child Health, Department of Surgery, and Liver Unit, King's College Hospital \\ Medical School, London
}

SUMMARY Of 47 consecutive infants with extrahepatic biliary atresia, effective bile drainage with the return of the serum bilirubin concentration to normal, was achieved in $17(38 \%)$. Direct bile duct-to-bowel anastomosis, attempted in 15 infants, produced bile drainage in only those 4 $(9 \%)$ in whom bile could be seen within the bile duct remnants at laparotomy. $13(45 \%)$ of 29 infants subjected to portoenterostomy (direct liver-to-bowel anastomosis) had satisfactory prolonged bile drainage with normal serum bilirubin values. Although a correct preoperative diagnosis was made in each case, in $3(6 \%)$ the 72 -hour faecal rose bengal ${ }^{131}$ I excretion was greater than $10 \%$ of the injected dose, and in $5(11 \%)$ the hepatic histology did not indicate bile duct obstruction, showing that both investigations are necessary for preoperative diagnosis. Preoperative clinical, laboratory, and hepatic histological features in the 16 jaundice-free survivors showed no significant difference when compared with the 31 infants in whom surgery was successful. Cholangitis occurred in only $7(43 \%)$ of 16 infants with satisfactory bile drainage and was easily controlled with antibiotic treatment. No cutaneous enterostomies were performed. In most survivors liver function tests remain abnormal, but the patients are symptom-free. While it is too early to predict a long-term prognosis for these children, our eldest survivors are healthy and show normal development.

Extrahepatic biliary atresia is characterised by complete obstruction of bile flow due to obliteration, or complete destruction, of part or all of the extrahepatic bile ducts. There is associated intrahepatic fibrosis which leads ultimately to biliary cirrhosis. Without effective surgery death from cirrhosis and its complications occurs in most children by age 2 years.

Results of surgical treatment of biliary atresia vary greatly. ${ }^{1}$ However, reports from two centres in Japan and North America, each with a wide experience of the condition, indicate that jaundice-free survival has been achieved after portoenterostomy in $42 \%^{2}$ and $55 \%^{3}$ of children with so-called 'noncorrectable' type of atresia which extends into the proximal bile ducts in the porta hepatis. Surprisingly, only $17 \%$ success was achieved by the more conventional technique of anastomosing residual segments of proximal bile duct to bowel. ${ }^{3}$

This report describes the results of surgical treatment in 47 consecutive children referred with extrahepatic biliary atresia in the last 5 years and considers the influence of preoperative factors, laparotomy findings, surgical procedures, and subsequent complications on the short-term prognosis.

\section{Patients and methods}

From July 1973 to December 1978, extrahepatic biliary atresia was confirmed at laparotomy in 47 patients, 25 girls and 22 boys. Genetic causes of cholestasis had been excluded by appropriate testing. ${ }^{4}$ The ${ }^{131}$ I rose bengal faecal excretion was less than $10 \%$ of the injected dose in a 72 -hour period, and percutaneous liver biopsy showed changes consistent with bile duct obstruction, in all except the cases specified below. Biopsies were obtained using the Menghini technique with local anaesthesia.

The histological findings in the liver biopsies were assessed prospectively by two observers (BDP and APM). Cirrhosis was graded as being absent, present, or doubtful; and 11 pathological features were graded as being absent, mild, moderate, or severe (Table 2). A histological diagnosis of bile 
duct obstruction was made when increased fibrosis, oedema, bile duct reduplication, and cellular infiltrate were present in all portal tracts in the biopsy. The diagnosis was considered definite, probable, or unlikely, depending on the severity of such changes. Standard laboratory methods were used to determine serum bilirubin, aspartate amino-transferase, alkaline phosphatase, gamma-glutamyl transpeptidase, albumin, and prothrombin time. Data were analysed by computer using standard statistical techniques.

For purposes of comparison the 16 patients who survived without jaundice were designated group 1, the remaining 31 cases group 2 .

\section{Results}

Preoperative clinical and laboratory features. All infants were born to healthy unrelated parents from families without any history of significant liver disease. Pregnancies were of normal duration but the birthweights of 4 infants were below the 5th centile for gestational age. Major congenital anomalies-comprising colomboma of the left eye, bilateral cleft palate and lip, duodenal stenosis with malrotation in the bowel-were present in 3 infants in whom surgery was ineffective, and malrotation of the bowel was present in one infant in whom surgery was successful.

Jaundice dating from the first week of life occurred in 36 infants, but was first noticed after 4 weeks of age in 2, and in 1 at 12 weeks of age. Acholic stools were observed by the third week of life in most patients, but in 1 only at 12 weeks of age. Pruritus occurred in 9. Three infants had an episode of significant spontaneous bleeding.
The age on admission to this hospital ranged from 4 to 34 (mean 9.7) weeks. Hepatomegaly (liver edge palpable more than $3 \mathrm{~cm}$ below the costal margin) was noticed in 39 infants, and 22 had splenomegaly. An umbilical hernia was found in 8 infants. Two had hydroceles and 2 inguinal herniae, but none had ascites.

Significant increases in both total and conjugated serum bilirubin, aspartate transaminase, and alkaline phosphatase were found in all cases, but the range of values was wide (Table 1). Serum albumin concentrations were normal in all instances. Prolongation of the prothrombin time in 3 infants was rapidly corrected by vitamin $\mathrm{K}$. Generalised aminoaciduria occurred in 13, none of whom showed a raised serum amino-acid concentration. Nine had nonglucose-reducing substances in urine samples. There were no significant differences between groups 1 and 2 with respect to clinical or laboratory findings, with the exception of the umbilical hernia which occurred only in patients in group 2 $(0 \cdot 2>P>0 \cdot 1)$.

Hepatic histology, rose bengal excretion, and indication for laparotomy. 45 of 47 biopsies showed intracellular cholestasis, which was moderate or severe in $79 \% .12(25 \%)$ cases also showed moderate or severe giant cell transformation of the hepatocytes. In the portal tract there were moderate or severe changes as follows: increased fibrosis $(79 \%)$, cellular infiltration $(79 \%)$, bile duct reduplication $(75 \%)$, and oedema (55\%) (Table 2).

Although giant cell transformation was more often present in group 1 patients and disruption of limiting plate was less common in group 2, within

Table 1 Clinical and laboratory data for 47 patients with extrahepatic biliary atresia

\begin{tabular}{|c|c|c|c|c|c|c|}
\hline & \multicolumn{3}{|c|}{$\begin{array}{l}\text { Group } 1(n=16), \text { normal serum bilirubin } \\
\text { after surgery }\end{array}$} & \multicolumn{3}{|c|}{ Group $2(n=31)$, surgery unsuccessful } \\
\hline & Range & Mean & $S D$ & Range & Mean & $S D$ \\
\hline Gestation (weeks) & $37-42$ & $39 \cdot 2$ & $1 \cdot 2$ & $37-42$ & $39 \cdot 7$ & $1 \cdot 4$ \\
\hline Birthweight (kg) & $2 \cdot 2-3 \cdot 9$ & $3 \cdot 22$ & 0.4 & $2 \cdot 5-4 \cdot 08$ & 3.08 & 0.6 \\
\hline Age at onset of jaundice (days) & $1-28$ & $6 \cdot 5$ & $8 \cdot 8$ & $1-90$ & $8 \cdot 2$ & 16 \\
\hline Age at onset of acholic stools (weeks) & $1-6$ & $2 \cdot 2$ & $1 \cdot 7$ & $1-12$ & $3 . \overline{4}$ & $3 \cdot 0$ \\
\hline Hepatomegaly $(\mathrm{cm})$ & $2-7$ & $4 \cdot 0$ & $1 \cdot 34$ & $2-8$ & $4 \cdot 4$ & $1 \cdot 8$ \\
\hline Splenomegaly & 9 cases & & & 14 cases & & \\
\hline Umbilical hernia & - & - & - & 8 & - & 一 \\
\hline \multicolumn{7}{|l|}{ Bilirubin $(\mu \mathrm{mol} / 1)$} \\
\hline (normal range $3.4-20.4$ ) & $95-330$ & 218 & 74 & $80-345$ & 210 & 58 \\
\hline \multicolumn{7}{|l|}{ Aspartate transaminase (IU/1) } \\
\hline (normal range $10-45$ ) & $54-580$ & 189 & 120 & $74-900$ & 215 & 184 \\
\hline \multicolumn{7}{|l|}{ Alkaline phosphatase (IU/1) } \\
\hline (normal range 60-250) & $123-1350$ & 444 & 362 & $98-800$ & 381 & 179 \\
\hline \multicolumn{7}{|l|}{$\operatorname{Albumin}(\mathrm{g} / 1)$} \\
\hline (normal range $35-50$ ) & $35-43$ & 39 & $2 \cdot 9$ & $35-49$ & $4 \cdot 0$ & $4 \cdot 0$ \\
\hline \multicolumn{7}{|l|}{ Rose-bengal } \\
\hline ( $\%$ faecal excretion) & $2-10$ & $5 \cdot 0$ & $2 \cdot 6$ & $0-15$ & $3 \cdot 5$ & $3 \cdot 5$ \\
\hline Age at operation (weeks) & $8-21$ & $12 \cdot 1$ & $4 \cdot 1$ & 6-37 & $13 \cdot 8$ & $6 \cdot 3$ \\
\hline
\end{tabular}

Conversion: SI to traditional units-bilirubin: $1 \mu \mathrm{mol} / 1 \approx 0.058 \mathrm{mg} / 100 \mathrm{ml}$, albumin: $1 \mathrm{~g} / 1 \approx 0 \cdot 1 \mathrm{~g} / 100 \mathrm{ml}$. 
Table 2 Histological assessment of percutaneous preoperative liver biopsy in patients free from jaundice (group 1) and with continuing jaundice (group 2)

\begin{tabular}{|c|c|c|c|c|c|}
\hline Cirrhosis & & Doubtfu & & Definite & \\
\hline $\begin{array}{l}\text { Group } 1 \\
\text { Group } 2\end{array}$ & & $\begin{array}{r}4 \\
10\end{array}$ & $\begin{array}{l}1 \\
2\end{array}$ & & \\
\hline Portal tract & Group & None & Mild & Moderate & Severe \\
\hline \multirow[t]{2}{*}{ Increased fibrosis } & 1 & 0 & 2 & 12 & 2 \\
\hline & $\begin{array}{l}2 \\
1\end{array}$ & $\begin{array}{l}3 \\
1\end{array}$ & $\begin{array}{l}5 \\
6\end{array}$ & $\begin{array}{r}21 \\
9\end{array}$ & $\begin{array}{l}2 \\
0\end{array}$ \\
\hline Oedema & 2 & 2 & 12 & 16 & 1 \\
\hline \multirow[t]{2}{*}{ Cellular infiltrate } & 1 & $\mathbf{0}$ & 6 & 10 & - \\
\hline & 2 & 1 & 17 & 13 & - \\
\hline \multirow[t]{2}{*}{ Bile duct reduplication } & 1 & $\mathbf{0}$ & 7 & 7 & 2 \\
\hline & 2 & 3 & 10 & 14 & 4 \\
\hline \multirow[t]{2}{*}{ Bile plugs } & 1 & 8 & 5 & 2 & 1 \\
\hline & 2 & 11 & 14 & 6 & 0 \\
\hline $\begin{array}{l}\text { Disruption of } \\
\text { limiting plates }\end{array}$ & $\begin{array}{l}1 \\
2\end{array}$ & $\begin{array}{l}1 \\
9\end{array}$ & $\begin{array}{l}15 \\
20\end{array}$ & $\begin{array}{l}0 \\
2\end{array}$ & $\begin{array}{l}\mathbf{0} \\
\mathbf{0}\end{array}$ \\
\hline \multicolumn{6}{|l|}{ Parenchyma } \\
\hline Intralobular fibrosis & $\begin{array}{l}1 \\
2\end{array}$ & $\begin{array}{r}5 \\
13\end{array}$ & $\begin{array}{l}11 \\
17\end{array}$ & $\begin{array}{l}0 \\
1\end{array}$ & $\begin{array}{l}\mathbf{0} \\
\mathbf{0}\end{array}$ \\
\hline Hepatic necrosis & $\begin{array}{l}1 \\
2\end{array}$ & $\begin{array}{r}4 \\
12\end{array}$ & $\begin{array}{l}12 \\
18\end{array}$ & $\begin{array}{l}0 \\
1\end{array}$ & $\begin{array}{l}0 \\
0\end{array}$ \\
\hline \multirow{2}{*}{$\begin{array}{l}\text { Giant cells } \\
\text { transformation }\end{array}$} & & & & & \\
\hline & $\begin{array}{l}1 \\
2\end{array}$ & $\begin{array}{r}2 \\
12\end{array}$ & $\begin{array}{l}10 \\
11\end{array}$ & $\begin{array}{l}1 \\
8\end{array}$ & $\begin{array}{l}3 \\
0\end{array}$ \\
\hline \multirow[t]{2}{*}{ Cellular infiltrate } & 1 & $\mathbf{0}$ & 13 & 3 & 0 \\
\hline & 2 & $\mathbf{0}$ & 28 & 3 & $\mathbf{0}$ \\
\hline Cholestasis & 1 & 1 & 2 & 11 & 2 \\
\hline
\end{tabular}

both groups all grades of severity were observed for the other features listed in Table 2. Statistical analysis showed a correlation between the age at biopsy and interstitial fibrosis $(\mathrm{P}<0.0015)$ and cirrhosis $(\mathrm{P}<0 \cdot 01)$, but not for other histological features. There was no correlation between the age at biopsy and the confidence of the histological diagnosis.

In 18 cases a firm histological diagnosis of bile duct obstruction was made, while in 24 cases the diagnosis was considered to be probable. In 5 infants portal tract changes were mild or absent and bile duct obstruction was considered unlikely. In all 5 however, the rose bengal excretion was less than $10 \%$, and laparotomy was advised because hepatocellular changes were only mild or moderate. The ${ }^{131}$ I rose bengal faecal excretion was less than $10 \%$ in all except 3 infants, in whom the excretion was $10 \cdot 3,11.4$, and $15 \%$. In the third case there was urinary contamination of the stools. Hepatic histology indicated bile duct obstruction in each.

Laparotomy at age 10 weeks was undertaken in one case because the liver biopsy was strongly indicative of bile duct obstruction and the rose bengal excretion was $9 \%$, but operative cholangiography showed a patent narrow biliary tree. Repeat laparotomy at age 16 weeks when the rose bengal faecal excretion was $4 \%$ showed biliary atresia extending to the common hepatic duct. A bile duct to jejunal anastomosis was unsuccessful.

Laparotomy findings, operative procedures, and results. All operations were performed by one surgeon (E R H). 20 children had cirrhosis, 8 being in group 1.12 (6 in each group) had an increase in free fluid. Operative procedures and their outcomes are shown (Fig. 1). In 15 children, atresia of the common bile ducts and distal hepatic ducts with a macroscopically patent segment extending up to the liver was found. Each was treated by anastomising the patent proximal segment of bile duct to a $30 \mathrm{~cm}$ Roux-en-Y loop of jejunum, but in only those 4 in whom bile was identified in the patent bile duct was this operation successful in producing bile drainage, as shown by bile-pigmented stools and a reduction in the serum bilirubin concentration to normal. In one of these, intractable diarrhoea developed 3 months after surgery, leading to death 9 months later. A subphrenic abscess was found at necropsy. Three survive with normal concentrations of serum bilirubin, 3,4 , and 33 months after surgery. Nine died of cirrhosis, the age of death ranging from 3 to 24 (mean $10 \cdot 7$, SE $2 \cdot 0$ ) months.

There were 2 early postoperative deaths due to ileus and peritonitis, 4 and 62 days after surgery.

In 32 infants the atretic process extended up to the porta hepatis and there were no macroscopically visible patent bile ducts. In 3 patients in whom no bile duct remnants were seen and advanced cirrhosis and portal hypertension were present, no corrective operative procedure was attempted. 29 cases were treated by portoenterostomy with a technique similar to that described by Kasai. ${ }^{5-7}$ In this operation the remnants of the extrahepatic ducts were identified by dissecting the gall bladder and cystic duct towards the porta hepatis. The duct remnants were dissected completely to their origin

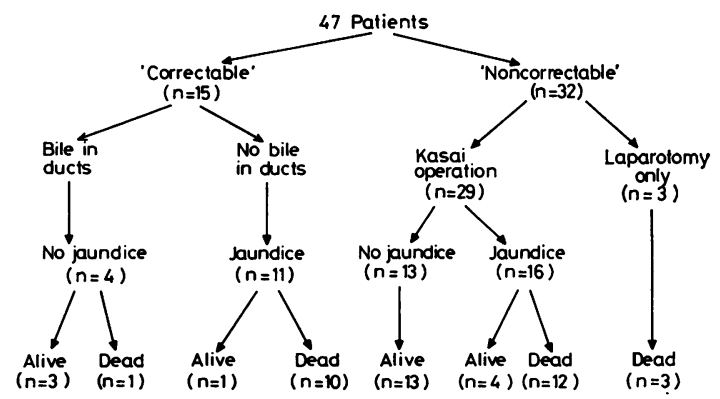

Fig. 1 Summary of operative diagnosis and outcome of surgical treatment in 47 consecutive cases of extrahepatic biliary atresia. 
above the bifurcation of the portal vein. The remnants were transected at the junction with the liver capsule and an anastomosis constructed between the edges of the transected tissue and a $30 \mathrm{~cm}$ Roux-en-Y loop of jejunum using fine, interrupted sutures of silk. Cutaneous enterostomies were not used in any operation.

Hepato-portoenterostomy was ineffective in returning the serum bilirubin concentration to normal in 16 patients. In 2 of these the stools became pigmented and the serum bilirubin fell significantly. One developed ascites and died of liver failure within 3 months. The other infant died unexpectedly in her sleep 2 months after surgery, the last recorded total serum bilirubin concentration being $94 \mu \mathrm{mol} / \mathrm{l}$ $(5.5 \mathrm{mg} / 100 \mathrm{ml}) .14$ have already died from cirrhosis, the age of death ranging from 4 to 44 (mean 14, SE 3) months. The remaining 13 infants have survived without jaundice for 8 to 65 (mean 20, SE 3.5) months after surgery. Bile-stained stools became apparent in the first postoperative week in 5 children, and in the remainder between the 2 nd and 15th (mean 3.6) week. The serum bilirubin concentrations returned to normal 1 to 26 (mean $11 \cdot 5$ ) weeks after surgery.

With both operations all infants have received prophylactic antibiotics, generally in the form of a cephalosporin or intravenous gentamicin for 1 to 4 weeks after surgery. While on milk feeds a supplement of medium-chain triglycerides was used. A multi-vitamin preparation (Ketovite) was given orally. Intramuscular vitamin $\mathrm{K}, 5 \mathrm{mg} /$ weekly and vitamin D, $10000 \mathrm{IU} /$ monthly, were given for the first 3 months after surgery. All infants received $4 \mathrm{~g}$ cholestyramine a day in divided doses with feeds, and phenobarbitone $15-45 \mathrm{mg}$ each evening.

All 16 survivors show normal development for their ages (range 3-65) mean 20, SE 4.5 months. Their growth is satisfactory with weights ranging from the 3rd to 75 th centile, the mean being at the 35 th centile. None has any symptom or signsuch as rickets-attributable to hepatobiliary disease. 14 have easily palpable, firm or hard livers, and 13 have persistently palpable spleens. In one child a previously palpable spleen can no longer be detected.

Although the serum bilirubin values have returned to normal, the aspartate transaminase (Fig. 2) and alkaline phosphatase (Fig. 3) are abnormal in most patients. Serum albumin and prothrombin concentrations are normal.

Complications. A clinical diagnosis of cholangitis was made in patients who developed unexplained fever associated with a rise in serum bilirubin concentration. In only 2 cases was a pathogen (Escher-

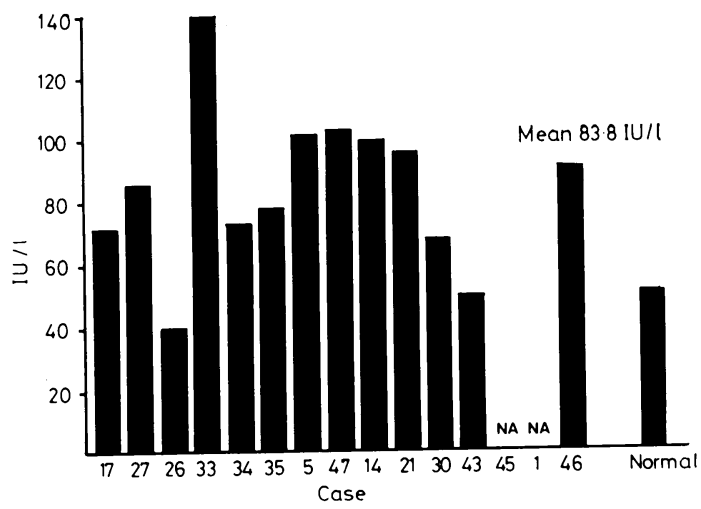

Fig. 2 Aspartate transaminase concentration at last follow-up in jaundice-free survivors.

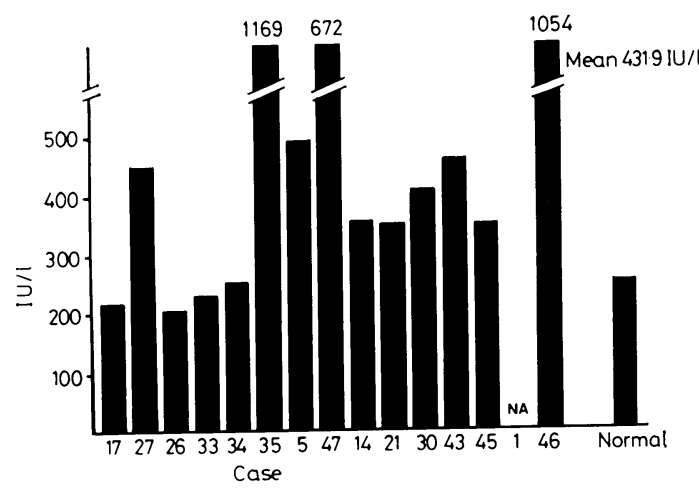

Fig. 3 Alkaline phosphatase concentration at most recent follow-up of jaundice-free survivors.

chia coli) found on blood culture and in one on liver biopsy culture. Twelve such episodes occurred in 7 infants, 3 infants had two attacks, and 1 infant three. In every instance, the cholangitis settled rapidly after intravenous antibiotics in the form of ampicillin (6 cases), gentamicin (5 cases), and cotrimoxazole ( 1 case). One episode occurred on the 10th postoperative day, ten between 1 and 6 months after surgery, and one after 10 months. Four episodes occurred in infants receiving cephalosporin prophylaxis. Ascites occurred in 7 children between 2 and 19 months after surgery. This was controlled by spironolactone in a dose of $25-100 \mathrm{mg} / 24$ hours which was stopped without recurrence of ascites after 4 to 12 weeks in all but 3 cases.

In only one child was alimentary bleeding a problem. Oesophageal transection was performed at age 40 months because of recurrent haematemesis. No further bleeding has taken place in the subsequent 3 years. 


\section{Discussion}

This report shows that the operation of portoenterostomy can result in jaundice-free survival for children with extrahepatic biliary atresia who had previously been thought to be 'inoperable'. It also calls into question the use of the term 'correctable' as applied to extrahepatic biliary atresia. 'Correctable', in this context, implies the presence of residual segments of extrahepatic bile ducts in the porta hepatis which communicate with intrahepatic ducts and through which bile drainage may occur after the construction of a conventional bile duct-to-bowel anastomosis. In previous series of extrahepatic biliary atresia, between 4 and $35 \%$ of cases were considered 'correctable', ${ }^{1}$ but effective long-term bile drainage is exceptional. ${ }^{8}$ In this series 'correctable' atresia was overdiagnosed at laparotomy and of 15 in whom a bile duct-to-bowel anastomosis was made, only 4 who were found to have bile in the bile duct remnants at operation lost their jaundice. Thus only $8.5 \%$ of 47 cases were 'correctable'. We now believe that when bile is not found in these residual segments, the more radical operation of portoenterostomy should be performed, as $13(45 \%)$ of 29 cases diagnosed as 'noncorrectable' lost their jaundice after it. This result is similar to the $42 \%$ success of portoenterostomy described in a detailed report by Altman ${ }^{2}$ from a 5 -year exprrience of this operation, and to the $55 \%$ success achieved by Kasai et al. ${ }^{3}$ during a 6-year period.

Less spectacular results from portoenterostomy continue to appear however, and of 111 cases treated in this way by Odièvre, ${ }^{9}$ only $22(19 \%)$ were free of jaundice one year later.

Reports from Japan ${ }^{3} 10$ indicate that best results are achieved if portoenterostomy is performed before the child is aged 60 days. For example, Kasai et al. obtained $91 \%$ bile drainage in patients younger than 60 days, but only $17 \%$ in children over 90 days of age. ${ }^{3}$ The results in this series show a similar trend but to a less pronounced degree, for although $8(47 \%)$ of 17 of those operated on at less than 12 weeks became free of jaundice, $5(41 \%)$ of 12 operated on after 12 weeks were equally successful. Laparotomy therefore, should not be denied to older infants but should be performed as soon as biliary atresia is suspected. The value of the combined use of liver biopsy and the ${ }^{131}$ I rose bengal faecal excretion in prelaparotomy diagnosis is well illustrated in this series. A faecal excretion in 72 hours of less than $10 \%$ of the intravenously injected rose bengal was found in $44(93 \%)$, and in 42 $(89 \%)$ biopsies was considered indicative of extrahepatic biliary atresia, but when considered together, severe cholestasis was correctly attributed to extrahepatic biliary atresia in each instance.

The results of surgery did not correlate with the preoperative clinical or laboratory features or with the severity of liver damage found in preoperative liver biopsies, either in this series or in that of Hitch et al. ${ }^{11}$ Effective bile drainage was achieved in 8 infants with cirrhosis at laparotomy.

Ascending cholangitis is often a problem after the successful construction of any biliary-enteric anastomosis. In patients with extrahepatic biliary atresia, it may cause considerable morbidity and add to the liver damage. Diagnostic criteria for cholangitis vary but the reported incidence ranges from $71 \%{ }^{12}$ to as high as $100 \%{ }^{13}$ despite complex surgical procedures-including the fashioning of a cutaneous biliary fistula-undertaken to prevent cholangitis. Despite using criteria which would seem to overdiagnose cholangitis, the overall incidence was only $43 \%$ with the use of a simple Roux-en-Y drainage loop. Although 4 children had repeated attacks, all settled rapidly with systemic gentamicin. The mechanism of cholangitis is poorly understood, while contamination by gastrointestinal organisms and ascending infection is favoured as a cause, ${ }^{\mathbf{1 4}}$ Hirsig et al. ${ }^{15}$ suggest that obstruction to hepatic lymphatic drainage is an important factor.

Portal hypertension is another consequence of biliary atresia frequently found in long-term survivors-over half of cases of Miyata et al..$^{12}$ and of Alagille ${ }^{16}$-but it may resolve spontaneously. ${ }^{9}$ Only one child in this series had problems from portal hypertension requiring oesophageal transection for severe bleeding at $\mathbf{4 0}$ months of age.

Most children in whom surgery was successful are thriving and symptom-free, in marked contrast to those in whom surgery was unsuccessful most had clinically hard livers and splenomegaly. The serum alkaline phosphatase levels have returned to normal in only 4 of 14 , while aspartate transaminase is normal in only 2 of 13 . Further follow-up will be necessary to determine the ultimate prognosis for this group of infants. On-going liver disease maybe as important as effective bile drainage in determining long-term survival. Follow-up liver biopsies in 8 of 11 jaundice-free survivors after portoenterostomy showed progression of hepatic fibrosis. ${ }^{17}$ This may occur without attacks of cholangitis. ${ }^{18}$ The mechanisms of the progressive liver damage and its course are unknown and unpredictable. Healthy long-term survival to the third decade does occur after portoenterostomy ${ }^{3}$ and $36 \%$ of a series of cases operated on by 60 days of age are alive at 5 years. ${ }^{19}$ Portoenterostomy therefore, although not an ideal operation, currently offers the best hope for children with extrahepatic biliary atresia who had 
previously been considered 'inoperable'. Earlier diagnosis and improvements in surgical technique may allow more infants to benefit, but better understanding and control of the intrahepatic complications or associations of the atretic process would appear to be necessary if all are to benefit from surgery.

We thank our colleagues in the Division of Pathology, particularly Mr M Clarke and Dr Barrett of the Department of Nuclear Medicine, for assistance in the care of these patients, the nursing staff who supervised the rose bengal faecal excretion tests, Dr G Psacharopoulos for statistical analysis, Mrs Pamela Golding for typing the manuscript, and the many colleagues who have referred patients and assisted in their follow-up.

H T P was supported by a grant from the Cystic Fibrosis Research Trust.

\section{References}

1 Howard E R, Mowat A P. Extrahepatic biliary atresia. Recent developments in management. Arch Dis Child $1977 ; 52: 825-7$.

2 Altman R P. The porto-enterostomy procedure for biliary atresia: a five-year experience. Ann Surg 1978; 188: 351-62.

3 Kasai M, Suzuki H, Ohashi E, Ohi R, Chiba T, Okamoto A. Technique and results of operative management in biliary atresia. World J Surg 1978; 2: 571-80.

4 Mowat A P. Liver disorders in childhood. London: Butterworth, 1979.

5 Kasai M, Suzuki S. A new operation for non-correctable biliary atresia; hepatic portoenterostomy. Shujutsu 1959; 13: 733-9.

6 Kimura K, Tsugawa C, Kubo M, Matsumoto Y, Itoh H. Technical aspects of hepatic portal dissection in biliary atresia. J Ped Surg 1979; 14: 27-32.

7 Howard E R. Extrahepatic biliary atresia. In: Maingot
R, ed. Abdominal operations, ninth edition. New York: Appleton-Century-Crofts, 1979: 2729-48.

8 Berenson M M, Garde E R, Moody F G. 25-year survival after surgery for complete extrahepatic biliary atresia. Gastroenterology 1974; 66: 260-3.

9 Odièvre $M$. Long-term results of surgical treatment of biliary atresia. World J Surg 1978; 2: 589-94.

10 Arima E, Kikuchi J, Sakoguchi Y, Akita K. Surgicallycorrectable biliary atresia: a follow-up study. Acta Med Univ Kagoshima 1976; 18: 39-45.

11 Hitch D C, Shikes R H, Lilly J R. Determinance of survival after Kasai's operation for biliary atresia using actuarial analysis. $J$ Pediatr Surg 1979; 14: 310-4.

12 Miyata M, Satani M, Ueda T, Okamoto E. Long-term results of hepatic portoenterostomy for biliary atresia: special reference to post-operative portal hypertension. Surgery 1974; 76: 234-7.

13 Lilly J R, Hitch D C. Post-operative descending cholangitis following portoenterostomy for biliary atresia. Measures for control. World J Surg 1978; 2: 581-7.

14 Hitch D C, Lilly J R. Identification, quantification, and significance of bacterial growth in the biliary tract after Kasai's operation. J Pediatr Surg 1978; 13: 563-9.

15 Hirsig J, Kara O, Rickman P P. Experimental investigation into the aetiology of cholangitis following operation for biliary atresia. J Pediatr Surg 1978; 13: 55-7.

16 Alagille D. Long-term results of hepatic portoenterostomy. In: Jauitt N B, ed. Neonatal hepatitis and biliary atresia. NIH 79-1296. Washington DC: United States Department of Health, Education, and Welfare, 1979: 411-6.

17 Altman R P, Chandra R, Lilly J R. On-going cirrhosis after successful portico-enterostomy in infants with biliary atresia. J Pediatr Surg 1975; 10: 685-91.

18 Suruga K. Invited commentary on the results of surgery for biliary atresia. World J Surg 1978; 2: 593-4.

19 Sawaguchi S, Akiyama H, Nakajo T. Long-term follow up after radical operation for biliary atresia. In: Japan Medical Research Foundation, ed. Cholestasis in infancy. Tokyo: University of Tokyo Press, 1980: 371-80.

Correspondence to Dr A P Mowat, Department of Child Health, King's College Hospital Medical School, Denmark Hill, London SE5 8RX.

Received 19 September 1979 